\title{
Guidance for Implementing COVID-19 Prevention Strategies in the Context of Varying Community Transmission Levels and Vaccination Coverage
}

\author{
Athalia Christie, MIA ${ }^{1}$; John T. Brooks, MD ${ }^{1}$; Lauri A. Hicks, DO루 Erin K. Sauber-Schatz, PhD ${ }^{1}$; Jonathan S. Yoder, MSW, MPH ${ }^{1}$; \\ Margaret A. Honein, $\mathrm{PhD}^{1}$; CDC COVID-19 Response Team
}

\begin{abstract}
On July 27, 2021, this report was posted as an MMWR Early Release on the MMWR website (https://www.cdc.gov/mmwr).
\end{abstract}

COVID-19 vaccination remains the most effective means to achieve control of the pandemic. In the United States, COVID-19 cases and deaths have markedly declined since their peak in early January 2021, due in part to increased vaccination coverage (1). However, during June 19-July 23, 2021, COVID-19 cases increased approximately 300\% nationally, followed by increases in hospitalizations and deaths, driven by the highly transmissible B.1.617.2 (Delta) variant* of SARS-CoV-2, the virus that causes COVID-19. Available data indicate that the vaccines authorized in the United States (Pfizer-BioNTech, Moderna, and Janssen [Johnson \& Johnson]) offer high levels of protection against severe illness and death from infection with the Delta variant and other currently circulating variants of the virus (2). Despite widespread availability, vaccine uptake has slowed nationally with wide variation in coverage by state (range $=33.9 \%-67.2 \%$ ) and by county (range $=8.8 \%-89.0 \%) .^{\dagger}$ Unvaccinated persons, as well as persons with certain immunocompromising conditions (3), remain at substantial risk for infection, severe illness, and death, especially in areas where the level of SARS-CoV-2 community transmission is high. The Delta variant is more than two times as transmissible as the original strains circulating at the start of the pandemic and is causing large, rapid increases in infections, which could compromise the capacity of some local and regional health care systems to provide medical care for the communities they serve. Until vaccination coverage is high and community transmission is low, public health practitioners, as well as schools, businesses, and institutions (organizations) need to regularly assess the need for prevention strategies to avoid stressing health care capacity and imperiling adequate care for both COVID-19 and other non-COVID-19 conditions. CDC recommends five critical factors be considered to inform local decisionmaking: 1) level of SARS-CoV-2 community transmission; 2) health system capacity; 3) COVID-19 vaccination coverage; 4) capacity for early detection of increases in COVID-19 cases;

\footnotetext{
* Point-in-time information is available from CDC COVID Data Tracker. https:// covid.cdc.gov/covid-data-tracker/\#variant-proportions

$\dagger$ Persons are considered fully vaccinated if $\geq 2$ weeks have elapsed following receipt of the second dose in a 2-dose series of Moderna or Pfizer-BioNTech mRNA COVID-19 vaccine, or $\geq 2$ weeks following receipt of 1-dose of Janssen (Johnson \& Johnson) vaccine. Data are available from CDC COVID Data Tracker. https://covid.cdc.gov/covid-data-tracker
}

and 5) populations at increased risk for severe outcomes from COVID-19. Among strategies to prevent COVID-19, CDC recommends all unvaccinated persons wear masks in public indoor settings. Based on emerging evidence on the Delta variant (2), CDC also recommends that fully vaccinated persons wear masks in public indoor settings in areas of substantial or high transmission. Fully vaccinated persons might consider wearing a mask in public indoor settings, regardless of transmission level, if they or someone in their household is immunocompromised or is at increased risk for severe disease, or if someone in their household is unvaccinated (including children aged $<12$ years who are currently ineligible for vaccination).

The principal mode by which persons are infected with SARS-CoV-2 is through exposure to respiratory fluids carrying infectious virus. ${ }^{\S}$ The risk for SARS-CoV-2 transmission in outdoor settings is low $(4,5)$. CDC recommends that public health practitioners and organizations prioritize prevention strategies for indoor settings. No one strategy is sufficient to prevent transmission, and multiple interventions should be used concurrently to reduce the spread of disease (๑). Proven effective strategies against SARS-CoV-2 transmission, beyond vaccination, include using masks consistently and correctly $(7,8)$, maximizing ventilation both through dilution $(9,10)$ and filtration (11) of air, and maintaining physical distance and avoiding crowds $(12,13)$. Basic public health measures such as staying home when sick, handwashing, and regular cleaning of high-touch surfaces should also be encouraged.

\section{Level of SARS-CoV-2 Community Transmission}

A person's risk for SARS-CoV-2 infection is directly related to the risk for exposure to infectious persons, which is largely determined by the extent of SARS-CoV-2 circulation in the surrounding community. $\mathrm{CDC}$ recommends assessing the level of community transmission using, at a minimum, two metrics: new COVID-19 cases per 100,000 persons in the last 7 days and percentage of positive SARS-CoV-2 diagnostic nucleic acid amplification tests in the last 7 days. For each of these metrics, CDC classifies transmission values as low, moderate, substantial, or high (Table). If the values for each

\footnotetext{
${ }^{\$}$ https://www.cdc.gov/coronavirus/2019-ncov/science/science-briefs/sars-cov2-transmission.html

Shttps://www.cdc.gov/coronavirus/2019-ncov/science/science-briefs/ transmission_k_12_schools.html
} 


\begin{tabular}{|c|c|c|c|c|}
\hline \multirow[b]{2}{*}{ Indicator } & \multicolumn{4}{|c|}{ Transmission level } \\
\hline & Low & Moderate & Substantial & High \\
\hline New cases per 100,000 persons in the past 7 days* & $0-9.99$ & $10.00-49.99$ & $50.00-99.99$ & $\geq 100.00$ \\
\hline Percentage of positive nucleic acid amplification tests in the past 7 days ${ }^{\dagger}$ & $<5.00$ & $5.00-7.99$ & $8.00-9.99$ & $\geq 10.00$ \\
\hline
\end{tabular}

of these two metrics differ (e.g., one indicates moderate and the other low), then the higher of the two should be used for decision-making. CDC recommends the geographic unit of analysis be county or core-based statistical area. In rural areas with low population densities, multiple counties might need to be combined to increase available data so that reliable inferences can be made. The level of SARS-CoV-2 transmission for any given area can change rapidly and should be reassessed at least weekly to ensure that the necessary layered prevention strategies are in place. In areas of substantial or high transmission, $\mathrm{CDC}$ recommends community leaders encourage vaccination and universal masking in indoor public spaces in addition to other layered prevention strategies to prevent further spread. Updated community transmission levels, as well as other indicators related to COVID-19, are available by county and state at the online CDC COVID Data Tracker** and are already used by many public health departments.

\section{Health System Capacity}

Data on usage of clinical care resources to manage patients with COVID-19 reflect underlying community disease incidence and can signal when urgent implementation of layered prevention strategies might be necessary to prevent overloading the health care system. Strains on critical care capacity can increase COVID-19 mortality $(14,15)$ while decreasing the availability and use of health care resources for non-COVID-19 related medical care $(16,17)$. CDC recommends public health departments and health care institutions monitor the available number and fraction of staffed inpatient and intensive care unit beds and develop thresholds, based on local health care system usage and remaining capacity, that would trigger community-wide application of layered prevention strategies.

\section{COVID-19 Vaccination Coverage}

Monitoring vaccination coverage in communities and organizations is recommended by CDC to gauge progress, focus vaccination efforts on populations whose coverage is low, and inform the need for additional prevention strategies. As of

\footnotetext{
** https://covid.cdc.gov/covid-data-tracker/\#county-view
}

July 23, 2021, the proportion of the total U.S. population who is fully vaccinated is $48.9 \%{ }^{\dagger \dagger}$ Of the 2,945 (91.4\%) U.S. counties reporting, vaccination coverage is $<40 \%$ in $1,856(63.0 \%)$ and $40 \%-49.9 \%$ in $672(22.8 \%)$; only 417 (14.2\%) of counties reported $\geq 50 \%$ COVID-19 vaccination coverage. Primary vaccination efforts should be accelerated in counties with low vaccination coverage. Public health practitioners should work with clinicians and community partners to build confidence in the vaccine and ensure equitable access. $\$ \$$ Organizations should establish supportive policies, such as allowing workers to receive vaccines during work hours or to take paid leave to get vaccinated at a community site, as well as offering flexible, nonpunitive sick leave options for employees.

\section{Capacity for Early Detection of Increases in COVID-19 Cases}

Certain populations are at high risk for exposure to, and thereby infection with, SARS-CoV-2. Such populations are especially well-suited for sentinel monitoring efforts to detect the early introduction and spread of COVID-19, particularly in areas with low vaccination coverage or where layered prevention strategies are not in use. CDC considers the capacity to monitor COVID-19 incidence in the following populations particularly useful due to their high risk of exposure or severe illness: students and staff members of kindergarten-grade 12 schools and institutions of higher education, health care workers, residents and staff members of long-term care facilities, incarcerated persons, homeless persons, and workers in high-density work sites (18-23).

Serial screening testing is an effective method to monitor for the early introduction and spread of COVID-19 (G). Low case detection rates can help demonstrate the effectiveness of current prevention strategies, thereby reducing barriers for returning to in-person learning and work. Rising case detection rates can serve as an early warning signal that prevention strategies need to be strengthened or added in the facility and the broader community. In addition, strategic serial testing can help stop transmission by rapidly identifying asymptomatic cases, which are estimated

$\dagger \dagger$ CDC’s COVID Data Tracker accessed July 23, 2021. https://covid.cdc.gov/ covid-data-tracker/\#vaccinations

$\$ \$$ https://www.cdc.gov/vaccines/covid-19/vaccinate-with-confidence.html 
to be the source for at least $50 \%$ of SARS-CoV-2 transmission $(24,25)$. With rapid identification, infectious persons can be isolated and contact tracing and quarantine can be promptly initiated to control further SARS-CoV-2 transmission.

\section{Populations at Risk for Severe Outcomes from COVID-19}

CDC recommends additional prevention strategies to safeguard populations at highest risk for severe outcomes from COVID-19, particularly in the context of the highly transmissible Delta variant. Unvaccinated persons remain at risk for infection, severe illness, and death. Advanced age, pregnancy, and an increasingly well-defined set of underlying medical conditions increase the risk for serious outcomes from COVID-19 among unvaccinated persons. 99 In addition, long-standing systemic health and social inequities have put members of certain racial and ethnic minority groups at increased risk for serious illness and mortality from COVID-19. Persons taking immunosuppressive medications, persons with hematologic cancers, and hemodialysis patients, among others, have shown reduced immunologic responses to COVID-19 mRNA vaccination and might remain at increased risk for severe COVID-19 following vaccination (3). CDC recommends unvaccinated persons should continue following all prevention strategies, including wearing a mask, until they are fully vaccinated. Immunocompromised persons should continue to take all recommended precautions until advised otherwise by their health care provider. Although COVID-19 vaccines authorized in the United States remain effective against severe outcomes from SARS-CoV-2 infection, a small proportion of persons who are fully vaccinated may become infected. Emerging evidence suggests that fully vaccinated persons who do become infected with the Delta variant are at risk for transmitting it to others (2), (CDC COVID-19 Response Team, unpublished data, 2021); therefore, CDC also recommends that fully vaccinated persons wear a mask in public indoor settings in areas of substantial or high transmission, and consider wearing a mask regardless of transmission level if they or someone in their household is immunocompromised or at increased risk for severe disease, or if someone in their household is unvaccinated (including children aged $<12$ years who are currently ineligible for vaccination). Public health practitioners and organizations should consider the characteristics of their local or setting-specific populations when determining whether to strengthen or add layered prevention strategies not only for effective disease control, but also to protect those persons at greatest risk for severe illness or death.

\footnotetext{
99 https://www.cdc.gov/coronavirus/2019-ncov/science/science-briefs/ underlying-evidence-table.html
}

\section{Discussion}

The most important public health action to end the pandemic remains increasing vaccination coverage, which saves lives, prevents illness, and reduces the spread of COVID-19. Effective COVID-19 prevention strategies are well documented and can help reduce community transmission until high vaccination coverage is achieved (G). To maximize protection of the community, prevention strategies should be strengthened or added if transmission worsens. Prevention strategies should only be relaxed or lifted after several weeks of continuous sustained improvement in the level of community transmission. In areas with low or no SARS-CoV-2 transmission and with testing capacity in place to detect early introduction or increases in spread of the virus, layered prevention strategies might be removed one at a time while monitoring closely for any evidence that COVID-19 cases are increasing.

The widespread availability and administration of COVID-19 vaccines has changed the trajectory of the pandemic in the United States and significantly reduced hospitalization and mortality among vaccinated persons (1). Increasing the proportion of eligible persons who are vaccinated reduces the risk for substantial or high community-wide transmission, which in turn reduces the risk for the emergence of new variants that could have the potential to overcome vaccine-induced immunity. However, vaccination coverage varies across the United States, and transmission risk remains considerable in areas with low vaccination coverage. Decisions to add or remove effective prevention strategies should be based on local data and public health recommendations. The emergence of more transmissible SARS-CoV-2 variants, including Delta, increases the urgency to expand vaccination coverage and for public health agencies and other organizations to collaboratively monitor the status of the pandemic in their communities and continue to apply layered prevention strategies to minimize preventable illness and death.

\section{Acknowledgments}

Case Data Section, Vaccine Data Section, CDC COVID-19 Data, Analytics \& Visualization Task Force; Data Monitoring and Reporting Section, CDC COVID-19 Vaccine Task Force; Heidi Moline, CDC.

Corresponding author: Athalia Christie, akc9@cdc.gov.

${ }^{1}$ CDC COVID-19 Response Team.

All authors have completed and submitted the International Committee of Medical Journal Editors form for disclosure of potential conflicts of interest. No potential conflicts of interest were disclosed. 


\section{Summary}

What is already known about this topic?

COVID-19 vaccines authorized in the United States are effective against severe illness and death from SARS-CoV-2 infection; however, current U.S. coverage is uneven. Implementation of layered prevention strategies reduces SARS-CoV-2 transmission.

What is added by this report?

Given the spread of the highly transmissible Delta variant, local decision-makers should assess the following factors to inform the need for layered prevention strategies across a range of settings: level of SARS-CoV-2 community transmission, health system capacity, vaccination coverage, capacity for early detection of increases in COVID-19 cases, and populations at risk for severe outcomes from COVID-19.

What are the implications for public health practice?

Although increasing COVID-19 vaccination coverage remains the most effective means to achieve control of the pandemic, additional layered prevention strategies will be needed in the short-term to minimize preventable morbidity and mortality.

\section{References}

1. Christie A, Henley SJ, Mattocks L, et al. Decreases in COVID-19 cases, emergency department visits, hospital admissions, and deaths among older adults following the introduction of COVID-19 vaccine-United States, September 6, 2020-May 1, 2021. MMWR Morb Mortal Wkly Rep 2021;70:858-64. PMID:34111059 https://doi.org/10.15585/ mmwr.mm7023e2

2. CDC. Science brief: COVID-19 vaccines and vaccination. Atlanta, GA: US Department of Health and Human Services, CDC; 2021. https:// www.cdc.gov/coronavirus/2019-ncov/science/science-briefs/fullyvaccinated-people.html

3. CDC. Clinical considerations for COVID-19 vaccination. Atlanta, GA: US Department of Health and Human Services, CDC; 2021. https:// www.cdc.gov/vaccines/covid-19/clinical-considerations/index.html

4. Bulfone TC, Malekinejad M, Rutherford GW, Razani N. Outdoor transmission of SARS-CoV-2 and other respiratory viruses: a systematic review. J Infect Dis 2021;223:550-61. PMID:33249484 https://doi. org/10.1093/infdis/jiaa742

5. Razani N, Malekinejad M, Rutherford GW. Clarification regarding outdoor transmission of SARS-CoV-2 and other respiratory viruses, a systematic review. J Infect Dis 2021. Epub June 4, 2021. https://doi. org/10.1093/infdis/jiab298

6. Honein MA, Christie A, Rose DA, et al.; CDC COVID-19 Response Team. Summary of guidance for public health strategies to address high levels of community transmission of SARS-CoV-2 and related deaths, December 2020. MMWR Morb Mortal Wkly Rep 2020;69:1860-7. PMID:33301434 https://doi.org/10.15585/mmwr.mm6949e2

7. Brooks JT, Butler JC. Effectiveness of mask wearing to control community spread of SARS-CoV-2. JAMA 2021;325:998-9. PMID:33566056 https://doi.org/10.1001/jama.2021.1505

8. Tomshine JR, Dennis KD, Bruhnke RE, et al. Combined effects of masking and distance on aerosol exposure potential. Mayo Clin Proc 2021;96:1792-800. PMID:34218858 https://doi.org/10.1016/j. mayocp.2021.05.007

9. Blocken B, van Druenen T, Ricci A, et al. Ventilation and air cleaning to limit aerosol particle concentrations in a gym during the COVID-19 pandemic. Build Environ 2021;193:107659. PMID:33568882 https:// doi.org/10.1016/j.buildenv.2021.107659
10. Vassella CC, Koch J, Henzi A, et al. From spontaneous to strategic natural window ventilation: improving indoor air quality in Swiss schools. Int J Hyg Environ Health 2021;234:113746. PMID:33819800 https://doi.org/10.1016/j.ijheh.2021.113746

11. Lindsley WG, Derk RC, Coyle JP, et al. Efficacy of portable air cleaners and masking for reducing indoor exposure to simulated exhaled SARS-CoV-2 aerosols_-United States, 2021. MMWR Morb Mortal Wkly Rep 2021;70:972-6. PMID:34237047 https://doi.org/10.15585/ mmwr.mm7027e1

12. Gonçalves MR, Dos Reis RCP, Tólio RP, et al. Social distancing, mask use, and transmission of severe acute respiratory syndrome coronavirus 2, Brazil, April-June 2020. Emerg Infect Dis 2021;27:2135-43. PMID:34087090

13. Kwon S, Joshi AD, Lo CH, et al. Association of social distancing and face mask use with risk of COVID-19. Nat Commun 2021;12:3737. PMID:34145289 https://doi.org/10.1038/s41467-021-24115-7

14. Bravata DM, Perkins AJ, Myers LJ, et al. Association of intensive care unit patient load and demand with mortality rates in US Department of Veterans Affairs hospitals during the COVID-19 pandemic. JAMA Netw Open 2021;4:e2034266. PMID:33464319 https://doi. $\mathrm{org} / 10.1001 /$ jamanetworkopen.2020.34266

15. Karaca-Mandic P, Sen S, Georgiou A, Zhu Y, Basu A. Association of COVID-19-related hospital use and overall COVID-19 mortality in the USA. J Gen Intern Med 2020. Epub August 19, 2020. PMID:32815058

16. Czeisler MÉ, Marynak K, Clarke KEN, et al. Delay or avoidance of medical care because of COVID-19-related concerns-United States, June 2020. MMWR Morb Mortal Wkly Rep 2020;69:1250-7. PMID:32915166 https://doi.org/10.15585/mmwr.mm6936a4

17. Whaley CM, Pera MF, Cantor J, et al. Changes in health services use among commercially insured US populations during the COVID-19 pandemic. JAMA Netw Open 2020;3:e2024984. PMID:33151319 https://doi.org/10.1001/jamanetworkopen.2020.24984

18. Paltiel AD, Zheng A, Walensky RP. Assessment of SARS-CoV-2 screening strategies to permit the safe reopening of college campuses in the United States. JAMA Netw Open 2020;3:e2016818. PMID:32735339 https:// doi.org/10.1001/jamanetworkopen.2020.16818

19. Lanier WA, Babitz KD, Collingwood A, et al. COVID-19 testing to sustain in-person instruction and extracurricular activities in high schools-Utah, November 2020-March 2021. MMWR Morb Mortal Wkly Rep 2021;70:785-91. PMID:34043614 https://doi.org/10.15585/ mmwr.mm7021e2

20. Bagchi S, Mak J, Li Q, et al. Rates of COVID-19 among residents and staff members in nursing homes-United States, May 25-November 22, 2020. MMWR Morb Mortal Wkly Rep 2021;70:52-5. PMID:33444301 https://doi.org/10.15585/mmwr.mm7002e2

21. Wallace M, Hagan L, Curran KG, et al. COVID-19 in correctional and detention facilities - United States, February-April 2020. MMWR Morb Mortal Wkly Rep 2020;69:587-90. PMID:32407300 https://doi. org/10.15585/mmwr.mm6919e1

22. Mosites E, Parker EM, Clarke KEN, et al.; COVID-19 Homelessness Team. Assessment of SARS-CoV-2 infection prevalence in homeless shelters-four U.S. cities, March 27-April 15, 2020. MMWR Morb Mortal Wkly Rep 2020;69:521-2. PMID:32352957 https://doi. org/10.15585/mmwr.mm6917e1

23. Waltenburg MA, Victoroff T, Rose CE, et al.; COVID-19 Response Team. Update: COVID-19 among workers in meat and poultry processing facilities-United States, April-May 2020. MMWR Morb Mortal Wkly Rep 2020;69:887-92. PMID:32644986 https://doi. org/10.15585/mmwr.mm6927e2

24. Moghadas SM, Fitzpatrick MC, Sah P, et al. The implications of silent transmission for the control of COVID-19 outbreaks. Proc Natl Acad Sci U S A 2020;117:17513-5. PMID:32632012 https://doi.org/10.1073/ pnas. 2008373117

25. Johansson MA, Quandelacy TM, Kada S, et al. SARS-CoV-2 transmission from people without COVID-19 symptoms. JAMA Netw Open 2021;4:e2035057. PMID:33410879 https://doi.org/10.1001/jamanetworkopen.2020.35057 